\title{
Lamotrigine-Induced Drug Reaction with Eosinophilia and Systemic Symptoms (DRESS) Masquerading as A Systemic Autoimmune Process
}

\section{Viraj Shah $^{1 *} \mid$ Priya Patel $^{1} \mid$ Korey Ullrich ${ }^{2}$}

*Correspondence: Viraj Shah

Address: ${ }^{1}$ Internal Medicine Residency, Florida Atlantic University, Boca Raton, FL; ${ }^{2}$ Affiliate Clinical Professor, Florida Atlantic University, Boca Raton, FL

e-mail $\bowtie:$ vshah@health.fau.edu

Received: 03 September 2021; Accepted: 15 September 2021

Copyright: (C) 2021 Shah V. This is an open-access article distributed under the terms of the Creative Commons Attribution License, which permits unrestricted use, distribution, and reproduction in any medium, provided that the original work is properly cited.

\begin{abstract}
Drug reaction with eosinophilia and systemic symptoms (DRESS) is a syndrome that is the consequence of a hypersensitivity reaction associated with exposure to certain medications in a population of individuals with presumed genetic predisposition to certain drugs. It is a potentially life-threatening condition that presents with rash and multi-organ system involvement. While characteristic findings include eosinophilia, lymphocytosis, and lymphadenopathy (LAD), clinical and pathological features can vary significantly. Aside from the observed immune response to certain drugs, its pathogenesis also includes reactivation of latent Herpesviridae. This suggests that drug exposure alone may not elicit the syndrome if neither active or latent Herpesviridae are present, Drug-induced hypersensitivity syndrome (DIHS) is postulated to be a consequence of the interplay between drug exposure and viral infection (latent or otherwise). With a mortality rate of approximately $10 \%$, timely recognition and treatment is imperative. This end can only be accomplished through greater familiarity with the clinical features of DRESS/DIHS. We present a case report of a 35-year-old woman with fever, rash, and lymphadenopathy suffering from lamotrigine-associated DRESS/DIHS masquerading as a systemic autoimmune process.

Keywords: Drug Reaction, Eosinophilia, Systemic Symptoms, Drug-Induced Hypersensitivity Syndrome, Lamotrigine, Lamictal, Regiscar, Cervical Lymphadenopathy
\end{abstract}

\section{Introduction}

DRESS, or DIHS, represents both cutaneous and visceral manifestations of a hypersensitivity reaction to certain medications. It was first described in 1937 as exfoliative dermatitis following treatment with the antifungal sulfanilamide or related compounds (Myers et al., 1937). Since that time, a number of drugs have been implicated including but not limited to allopurinol, dapsone, phenytoin and other anticonvulsants (Shear and Spielberg, 1988). It has been presumed that coincident viral infection, or reactivation of latent Herpesviridae, also contributes to the pathogenesis of this syndrome (Bocquet et al., 1996). It is characterized by fever, lymphadenopathy, hematologic abnormalities, and multi-organ involvement. Onset tends to occur weeks after initiation of the offending drug and flares may continue to occur well after the drug has been discontinued. Due to a lack of specific features, the diagnosis is 
frequently overlooked in routine clinical practice. With a mortality rate of approximately $10 \%$, timely recognition and treatment is imperative. Criteria for diagnosis have been established by the collective work of the RegiSCAR group (Table 1) and a Japanese consensus group. We present a case report of a 35year-old female with lamotrigine-associated DRESS/DIHS who responded well to corticosteroids following cessation of lamotrigine use.

Table 1: RegiSCAR Scoring System for Classifying DRESS applied to the Patient (Husain et al., 2013).

\begin{tabular}{|c|c|c|c|}
\hline Symptoms and Laboratory Findings for DRESS & Scoring (points) For DRESS & Patient Result & Patient Score \\
\hline Fever $38.5^{\circ} \mathrm{C}$ & No/U: (-)1, Y: 0 & $38.8^{\circ} \mathrm{C}$ & 0 \\
\hline Lymphadenopathy & No/U: 0, Y: 1 & Yes & 1 \\
\hline $\begin{array}{l}\text { Eosinophilia } \\
\text { Eosinophils (per } \mu \mathrm{L} \text { ) } \\
\text { Eosinophils (\%), if WBC }<4000\end{array}$ & $\begin{array}{l}700-1500: 1, \geq 1500: 2 \\
\text { 10-19.9\%: } 1, \geq 20 \%: 2 \\
\text { * Max score }=2\end{array}$ & No & 0 \\
\hline Atypical Lymphocytes & No/U: 0, Y: 1 & Absent & 0 \\
\hline $\begin{array}{l}\text { Skin Rash } \\
\text {-Extent (\% body surface area) } \\
\text {-Rash suggesting DRESS } \\
\text {-Biopsy suggesting DRESS }\end{array}$ & $\begin{array}{l}\text { No/U: } 0,>50 \%: 1 \\
\text { No: (-)1, U: 0, Y: } 1 \\
\text { No: (-)1, Y/U: } 0 \\
\text { *Minimum score = }-2, \text { Max }=2\end{array}$ & $\begin{array}{l}>50 \% \\
\text { Yes } \\
\text { Unknown }\end{array}$ & $\begin{array}{l}1 \\
1 \\
0\end{array}$ \\
\hline $\begin{array}{l}\text { Organ Involvement } \\
\text {-Liver } \\
\text {-Kidney } \\
\text {-Lung } \\
\text {-Muscle/heart } \\
\text {-Pancreas } \\
\text {-Other }\end{array}$ & $\begin{array}{l}\text { No/U: } 0, Y: 1 \\
\text { No/U: } 0, Y: 1 \\
\text { No/U: 0, Y: } 1 \\
\text { No/U: 0, Y: } 1 \\
\text { No/U: 0, Y: } 1 \\
\text { No/U: 0, Y: } 1 \\
\text { *Max score }=2\end{array}$ & $\begin{array}{l}\text { Yes } \\
\text { No } \\
\text { No } \\
\text { No } \\
\text { No } \\
\text { No }\end{array}$ & $\begin{array}{l}1 \\
1\end{array}$ \\
\hline Resolution $\geq 15$ days & No/U: (-)1, Y: 0 & Yes & 0 \\
\hline $\begin{array}{l}\text { Evaluations of other potential causes } \\
\text {-Antinuclear antibody } \\
\text {-Blood culture } \\
\text {-HAV/HBV/HVC } \\
\text {-Chlamydia/mycoplasma } \\
\text { *If none positive and } \geq 3 \text { of above } \\
\text { negative }\end{array}$ & No: $0, Y: 1$ & $\begin{array}{l}\text { Negative } \\
\text { Negative } \\
\text { Negative } \\
\text { Negative }\end{array}$ & 1 \\
\hline
\end{tabular}

\section{Total Score: 6 (Definite case of DRESS)}

Final Score of < 2, no case; score of 2-3, possible case; score 4-5, probable case; score $>5$, definite case. Range of total score is -4 to 9. U: Unknown, Y: Yes

\section{Case Presentation}

A 35-year-old female with past medical history significant for depression and anxiety presented to our emergency department for evaluation of two weeks of subjective fevers and intermittent chills. She denied known sick contacts and said she was without other systemic symptoms. Her medications included trazodone $50 \mathrm{mg}$ daily, valproic acid $100 \mathrm{mg}$ daily, lamotrigine $50 \mathrm{mg}$ twice daily, and fluoxetine $30 \mathrm{mg}$ daily. Vital signs were stable on presentation and physical examination revealed a diffuse maculopapular rash affecting the face, trunk, and all extremities with sparing of the palms and soles. 
Right cervical LAD was also appreciated. Her laboratory results were significant for Aspartate Aminotransferase (AST) of 211 (NR <35 U/L), Alanine Transferase (ALT) of 255 (NR < 55 U/L), Alkaline Phosphatase (ALP) of 195 (NR < $140 \mathrm{U} / \mathrm{L}$ ) and serum creatinine of $1.5 \mathrm{mg} / \mathrm{dl}$ with Blood Urea Nitrogen of $25 \mathrm{mg} / \mathrm{dl}$. Urine analysis and right upper quadrant ultrasound were unremarkable for any acute findings. Rheumatology was consulted for input regarding concern of occult systemic autoimmune disease. Serologic testing for infectious etiologies included but were not limited to the following: Human Immunodeficiency Virus (HIV), Cytomegalovirus (CMV), Epstein-Barr Virus (EBV), Toxoplasmosis, viral Hepatitis (A, B, and C), Parvovirus B19, Bartonella, Brucella, RPR; all were negative. Heterophile antibody, rheumatoid factor, antinuclear antibody, QuantiFERON, and blood cultures were also negative. A surgical biopsy of the enlarged right cervical lymph node was performed. The histological findings included palisading neutrophils, histiocytes, lymphocytes, and necrosis without evidence of malignancy or infection. The findings, most notably the presence of neutrophils, were not entirely consistent with those seen in KFD, which is typically characterized by a predominance of histiocytes and absence of neutrophils.

Further questioning of the patient revealed that she had been started on lamotrigine 50 mg daily five weeks prior to her admission and that the dose had been increased to $50 \mathrm{mg}$ twice daily 3 weeks prior. Given the patient's negative workup, the temporal relationship between the initiation of lamotrigine and the onset of her symptoms, the lack of another identifiable trigger, and her score per diagnostic criteria developed by the Registry of Severe Cutaneous Adverse Reactions or RegiSCAR Group (Table 1), she was diagnosed with DRESS/DIHS. Lamotrigine was discontinued and she was started on prednisone $40 \mathrm{mg} /$ day with plan to taper over a 2-month course (Husain et al., 2013). She was subsequently discharged after resolution of symptoms and upon follow-up denied recurrence of symptoms.

\section{Discussion}

A severe cutaneous reaction that would eventually become known as DRESS was initially observed as an exfoliative dermatitis in patients receiving sulfanilamide treatment for gonorrhea or candida vulvovaginitis (Myers et al., 1937). It was then observed in association with exposure to phenytoin, among other anticonvulsants. There were also descriptions of erythema multiforme, exanthematous eruption, toxic epidermal necrolysis, and Stevens-Johnson syndrome (Singer and Wallace, 1986; Shear and Spielberg, 1988).

Ever since that time, many different terms were used to describe this clinical syndrome, with those terms primarily depending on the culprit drug, such as allopurinol hypersensitivity syndrome, sulfone 
syndrome, and anti-convulsant hypersensitivity syndrome. To limit confusion, Bocquet, et al. (1996) proposed the use of the term DRESS in hopes of providing a single umbrella term with a clear description of the nature of these exposure-related syndromes. DRESS/DIHS is a severe reaction following drug initiation that most commonly develops 3-6 weeks following initiation of therapy (Cho et al., 2017). Common features include rash, fever, lymphadenopathy, and hematologic abnormalities. Drugs commonly implicated include anticonvulsants such as phenytoin, carbamazepine, phenobarbital, valproic acid, and lamotrigine as well as allopurinol, minocycline, sulfasalazine, nevirapine, vancomycin, trimethoprim-sulfamethoxazole, and abacavir (Cacoub et al., 2011). DRESS/DIHS is relatively rare, with an incidence of approximately one case per 1,000-10,000 exposures (Chiou et al., 2008). The pathophysiology of DRESS/DIHS is not entirely understood, but it is believed that the interplay between viral reactivation, particularly EBV, CMV, and HHV-6, and immunologic response from regulatory T-cells is paramount in evolution of this disease.

Reactivation of latent Herpesviridae infection, specifically HHV-6, has been observed to be associated with not only more severe disease but also a protracted clinical course (Miyagawa et al., 2016). In patients with DRESS/DIHS, an association has been observed between the presence of higher viral load or antibody titers and poorer outcomes. Polymorphisms influencing the process of drug breakdown (e.g. $\mathrm{N}$-acetylation) and mitigation of toxic metabolites are speculated to have some role in increasing one's susceptibility to developing DRESS/DIHS when exposed to specific drugs.

Skin manifestations are prevalent and tend to occur early in the disease course as an eruption of a maculopapular rash covering the trunk and extremities. The rash can eventually coalesce to form large patches of confluent erythema and facial swelling often occurs. Facial edema is a hallmark feature of the disease. Such cutaneous manifestations are estimated to occur in up to 73-100\% of the patients (Cho et al., 2017). Erythroderma may eventually develop as rash typically involves greater than half of the body surface area. The lesions vary but many are infiltrative papules or plaques with purpuric features. These cutaneous lesions are often polymorphoric: maculopapular, urticarial, exfoliative, lichenoid, pustular, bullous, target-like, or eczematous. Desquamation tends to evolve around the time of resolution.

Both hematologic and organ system dysfunction have been observed. Eosinophilia and atypical lymphocytosis are the most common hematologic manifestation of DRESS syndrome. Liver injury is the most common type of end-organ damage and typically presents in a cholestatic pattern (Peyrière et al., 2006). Notably, hepatic injury can occur well before the onset of characteristic cutaneous lesions. While renal involvement is generally mild, there have been documented cases of severe interstitial nephritis and acute tubular necrosis ultimately leading to acute renal failure. The lungs are the third most involved organ. Patients may present with spectrum of conditions ranging from pleurisy and interstitial 
pneumonitis to acute respiratory distress syndrome (ARDS). Cardiac involvement is primarily limited to two common entities: hypersensitivity myocarditis and acute necrotizing eosinophilic myocarditis. Involvement of other organs, albeit less likely, can be encountered including the brain, pancreas, gastrointestinal tract, and spleen (Cacoub et al., 2011).

Some of the short and long-term consequences of DRESS/DIHS are mentioned in Table 2. Patients with DRESS often develop signs and symptoms as early as 3 weeks following initiation of the offending drug. When compared to other familiar cutaneous hypersensitivity reactions such as Steven-Johnson Syndrome, toxic epidermal necrolysis, and acute generalized exanthematous pustulosis, the latency period for DRESS is much longer. This fact alone could increase the likelihood of overlooking the diagnosis altogether. Further, DRESS/DIHS share clinical features common to a wide spectrum of systemic disorders which further complicates the goal of timely and accurate diagnosis. Cervical lymphadenopathy has been reported as a finding in Lamotrigine-associated DRESS/DIHS (Schlienger et al., 1998). Surgical biopsy can assist in identifying the etiology of cervical lymphadenopathy because many of the potential etiologies like infections, neoplasms, and inflammatory disorders have unique histopathology. Further, DRESS/DIHS is generally considered a clinical diagnosis. Several diagnostic criteria have been developed including those drafted by the RegiSCAR group (Table 1) and a Japanese consensus group.

Table 2: Short- and long-term sequelae of DRESS/DIHS
\begin{tabular}{|l|}
\hline Arthralgia, reactive arthritis, rheumatoid arthritis \\
\hline Autoimmune thyroiditis Colitis/enteropathy \\
\hline Cutaneous autoimmune disease \\
\hline Vitiligo, alopecia areata \\
\hline Diabetes mellitus \\
\hline Encephalitis \\
\hline Fulminant hepatic failure \\
\hline Hemolytic anemia \\
\hline Myocarditis \\
\hline Pneumonitis \\
\hline Renal failure \\
\hline Systemic lupus erythematosus \\
\hline Venous thrombosis \\
\hline
\end{tabular}

If suspected, the offending agent should be discontinued with immediate initiation of supportive care. The general consensus is that systemic corticosteroids are the mainstay treatment for patients with DRESS syndrome (Husain et al., 2013). A starting dose of prednisolone or an equivalent of 0.5-1.0 $\mathrm{mg} / \mathrm{kg} /$ day with a gradual tapering over 2-3 months is typically recommended. As anticipated, our patient experienced significant interval improvement upon cessation of Lamotrigine and administration 
of systemic steroids.

It should be noted that patients with DRESS/DIHS are at risk for systemic autoimmune sequelae which can appear months to years after resolution of acute systemic involvement, this necessitating longterm monitoring aimed at its detection and subsequent management.

\section{Conclusion}

DRESS/DIHS often presents as a multi-system disorder which can mimic a systemic autoimmune process. It can be distinguished from other diagnoses by history of new drug exposure in the weeks prior to onset of rash or systemic symptoms as well as the absence of serologic and histologic findings commonly observed in autoimmune disease. This case highlights the value of early rheumatological consultation in preventing morbidity and mortality.

\section{References}

Bocquet H, Bagot M, Roujeau JC. Drug-induced pseudolymphoma and drug hypersensitivity syndrome (Drug Rash with Eosinophilia and Systemic Symptoms: DRESS). Semin Cutan Med Surg 1996; 15: 250-257.

Cacoub P, Musette P, Descamps V, Meyer O, Speirs C, Finzi L, Roujeau JC. The DRESS syndrome: a literature review. Am J Med 2011; 124: 588-597.

Chiou CC, Yang LC, Hung SI, Chang YC, Kuo TT, Ho HC, Hu S, Hong HS, Chung WH. Clinicopathological features and prognosis of drug rash with eosinophilia and systemic symptoms: a study of 30 cases in Taiwan. J Eur Acad Dermatol Venereol 2008; 22: 1044-1049.

Cho YT, Yang CW, Chu CY. Drug Reaction with Eosinophilia and Systemic Symptoms (DRESS): An Interplay among Drugs, Viruses, and Immune System. Int J Mol Sci 2017; 18: 1243.

Husain Z, Reddy BY, Schwartz RA. DRESS syndrome: Part II. Management and therapeutics. J Am Acad Dermatol 2013; 68: 709.e1-720.

Miyagawa F, Nakamura Y, Miyashita K, Iioka H, Himuro Y, Ogawa K, Nishimura C, Nishikawa M, Mitsui Y, Ito Y, Ommori R. Preferential expression of CD134, an HHV-6 cellular receptor, on CD4T cells in drug-induced hypersensitivity syndrome (DIHS)/drug reaction with eosinophilia and systemic symptoms (DRESS). J Dermatol Sci 2016; 83: 151-154.

Myers GB, Heide ECV, Balcerski M. Exfoliative Dermatitis Following Sulfanilamide. JAMA 1937; 109: 19831984.

Peyriere H, Dereure O, Breton H, Demoly P, Cociglio M, Blayac JP, Hillaire-Buys D, Network of the French Pharmacovigilance Centers. Variability in the clinical pattern of cutaneous side-effects of drugs with systemic symptoms: does a DRESS syndrome really exist? Br J Dermatol 2006; 155: 422-428.

Schlienger RG, Knowles SR, Shear NH. Lamotrigine-associated anticonvulsant hypersensitivity syndrome. Neurology 1998; 51: 1172-1175. 
Shear NH and Spielberg SP. Anticonvulsant hypersensitivity syndrome. In vitro assessment of risk. J Clin Invest 1988; 82: 1826-1832.

Singer JZ and Wallace SL. The allopurinol hypersensitivity syndrome. Unnecessary morbidity and mortality. Arthritis Rheum 1986; 29: 82-87. 